\title{
1 A First-Principles Scheme for Calculating the Electronic Structure of Strongly Correlated Materials: GW+DMFT
}

\author{
${ }^{a}$ F. Aryasetiawan ${ }^{1},{ }^{b}$ S. Biermann, ${ }^{c}$ A. Georges \\ ${ }^{a}$ Research Institute for Computational Sciences, AIST, 1-1-1 Umezono, Tsukuba Central 2, \\ Ibaraki 305-8568, Japan, \\ ${ }^{b}$ Centre de Physique Theorique, Ecole Polytechnique, 91128 Palaiseau, France \\ ${ }^{c}$ Laboratoire de Physique Theorique de 1'Ecole Normale Superieure 24, rue Lhomond 75231 \\ Paris Cedex 05 France
}

\subsection{Introduction}

The last few decades have witnessed a substantial progress in the field of electronic structure of materials. Using density functional theory (DFT) [22 25] within the local density approximation (LDA) or generalized gradient approximation (GGA) [38] it is quite a routine to calculate the electronic structure of relatively complicated materials containing tens of atoms per unit cell. The success of LDA, however, is also accompanied by a number of serious problems. It was noticed very early on that when applied to calculate the band structures of s-p semiconductors and insulators, it was found that the band gaps are systematically underestimated by some tens percents. Apart from the too small gaps, the band dispersions are very reasonable. This remarkable property of the LDA is still waiting for an explanation since formally there is no theoretical justification for identifying the one-particle Kohn-Sham eigenvalues as quasiparticle energies observed in photoemission experiments. Applications to alkali metals also indicate some problems, albeit less serious. When the band dispersions are compared with photoemission data, they are found to be too wide by 10-30\%. Some many-body calculations of the electron gas [50,33], however, suggest that the band widths are actually widened compared with the free-electron values and that the LDA performs better than it is commonly believed. If this turned out to be true, photoemission data would presumably need a complete revision. In any case, the LDA errors in s-p metals are probably less significant compared to the band gap errors in semiconductors and insulators.

A much more serious problem of the LDA arises when it is applied to calculate the electronic structures of so-called "strongly correlated systems". We have to be more precise with what we mean by correlations. Even in the electron gas, correlation as conventionally defined is rather large. It is as large as exchange that the two almost cancel each other leaving the free-electron band essentially unchanged. Thus, it is more appropriate in our case to define correlation as anything beyond the LDA rather than anything beyond the Fock exchange since the former is usually our starting point in electronic structure calculations of solids.

\footnotetext{
${ }^{1}$ Corresponding author
} 
Strongly correlated systems are characterized by partially occupied localized orbitals such as found in transition metal oxides or $4 \mathrm{f}$ metals. Here the problem is often more of qualitative rather than quantitative nature. It is often found that the LDA predicts a transition metal oxide to be a metal whereas experimentally it is an antiferromagnetic insulator. To cite some examples, $\mathrm{LaMnO}_{3}$, famous for its colossal magnetoresistance, and $\mathrm{La}_{2} \mathrm{CuO}_{4}$, a well-known parent compound of high-temperature superconductors, are antiferromagnetic insulators but predicted to be metals by the LDA [39]. In cases where the LDA does predict the correct structure, it is legitimate to ask if the one-particle spectrum is also reproduced correctly. According to the currently accepted interpretation, transition metal oxides may be classified as charge-transfer insulators [18 42], which are characterized by the presence of occupied and unoccupied $3 \mathrm{~d}$ bands with the oxygen $2 \mathrm{p}$ band in between. The gap is then formed by the oxygen $2 \mathrm{p}$ and unoccupied $3 \mathrm{~d}$ bands, unlike the gap in LDA, which is formed by the $3 \mathrm{~d}$ states (Mott-Hubbard gap). A more appropriate interpretation is to say that the highest valence state is a charge-transfer state: During photoemission a hole is created in the transition metal site but due to the strong $3 \mathrm{~d}$ Coulomb repulsion it is energetically more favourable for the hole to hop to the oxygen site despite the cost in energy transfer. A number of experimental data, notably $2 \mathrm{p}$ core photoemission resonance, suggest that the charge-transfer picture is more appropriate to describe the electronic structure of transition metal oxides. And of course in the case of $4 \mathrm{f}$ metals, the LDA, being a one-particle theory, is totally incapable of yielding the incoherent part of the spectral function or satellite structures.

The difficulties encountered by the LDA discussed above have prompted a number of attempts at improving the LDA. Notable among these is the GW approximation (GWA), developed systematically by Hedin in the early sixties [20]. He showed that the self-energy can be formally expanded in powers of the screened interaction $W$, the lowest term being $i G W$, where $G$ is the Green function. Due to computational difficulties, for a long time the applications of the GWA were restricted to the electron gas. With the rapid progress in computer power, applications to realistic materials eventually became possible about two decades ago. Numerous applications to semiconductors and insulators reveal that in most cases the GWA [10, 12] removes a large fraction of the LDA band-gap error. Applications to alkalis show band narrowing from the LDA values and account for more than half of the LDA error (although controversy about this issue still remains [29]).

The success of the GWA in sp materials has prompted further applications to more strongly correlated systems. For this type of materials the GWA has been found to be less successful. For example, GW calculation on nickel [8] does reproduce the photoemission quasiparticle band structure rather well, as compared with the LDA one where the $3 \mathrm{~d}$ band width is too large by about $1 \mathrm{eV}$, but the too large LDA exchange splitting of $0.6 \mathrm{eV}$ (experimentally 0.3 $\mathrm{eV}$ ) remains essentially unchanged. Moreover, the famous $6 \mathrm{eV}$ satellite is not reproduced. Application to $\mathrm{NiO}$ [9], a prototype of transition metal oxides, also reveals some shortcomings. One problem is related to the starting Green's function, usually constructed from the LDA Kohn-Sham orbitals and energies. In the LDA the band gap is very small, about $0.2 \mathrm{eV}$ compared with the $4 \mathrm{eV}$ experimental band gap. A commonly used procedure of performing a one-iteration $\mathrm{GW}$ calculation yields about $1 \mathrm{eV}$ gap, much too small. This problem is solved by performing a partial self-consistency, where knowledge of the self-energy from the previous iteration is used to construct a better starting one-particle Hamiltonian [9]. This procedure improves the band gap considerably to a self-consistent value of $5.5 \mathrm{eV}$ and at the 
same time increases the LDA magnetic moment from $0.9 \mu_{B}$ to about $1.6 \mu_{B}$ much closer to the experimental value of $1.8 \mu_{B}$. However, the GWA maintains the Mott-Hubbard gap, i.e., the gap is formed by the $3 \mathrm{~d}$ states as in the LDA, instead of the charge-transfer gap. In other words, the top of the valence band is dominated by the Ni 3d. A more recent calculation using a more refined procedure of partial self-consistency has also confirmed these results [16]. The problem with the GWA appears to arise from inadequate account of short-range correlations, probably not properly treated in the random-phase approximation (RPA).

Attempts at improving the LDA to treat strongly correlated systems were initiated by the $\mathrm{LDA}+\mathrm{U}$ method [3 4] 30, 5], which introduces on top of the LDA Hamiltonian a Hubbard U term and a double-counting correction term, usually applied to partially filled $3 \mathrm{~d}$ or $4 \mathrm{f}$ shells. The LDA+U method is essentially a Hartree-Fock approximation to the LDA+U Hamitonian. In the LDA, the Kohn-Sham potential does not explicitly distinguish between occupied and unoccupied orbitals so that they experience the same potential. In, for example, transition metal oxides, where the $3 \mathrm{~d}$ orbitals are partially occupied, this leads to metallicity or to underestimation of the band gap. The $\mathrm{LDA}+\mathrm{U}$ cures this problem by approximately pushing down the occupied orbitals by $U / 2$ and pushing up the unoccupied orbitals by $U / 2$, creating a lower and upper Hubbard band, thus opening up a gap of the order of the Hubbard $U$. The LDA+U method has been successfully applied to late transition metal oxides, rare earth compounds such as $\mathrm{CeSb}$, as well as to problems involving metal-insulator transition and charge-orbital ordering.

More recently, the idea of the LDA+U was extended further by treating the Hubbard $\mathrm{U}$ term in a more sophisticated fashion utilizing the dynamical mean-field theory (DMFT) [19]. The DMFT is remarkably well suited for treating systems with strong on-site correlations because the on-site electronic Coulomb interactions are summed to all orders. This is achieved by using a mapping onto a self-consistent quantum impurity problem, thereby including the effects of the surrounding in a mean-field approximation. The strength of the DMFT is its ability to properly describe Mott phenomenon or the formation of local moments, which is the key to understanding many physical properties in strongly correlated materials. The combination of LDA and DMFT takes advantage of the first-principles nature of LDA while at the same time incorporates local correlation effects not properly treated within the LDA. The LDA+DMFT method [7, 6, 31] has been successfully applied to a number of systems by now.

In both the LDA+U and LDA+DMFT methods, two fundamental problems remain unaddressed. First, the Hubbard U is usually treated as a parameter, and second, the Hubbard $\mathrm{U}$ term contains interaction already included in the LDA but it is not clear how to take into account this double-counting term in a precise way. Thus, a truly first-principles theory for treating strongly correlated systems is still lacking. In this article, we describe a dynamical mean-field approach for calculating the electronic structure of strongly correlated materials from first-principles [13 46]. The DMFT is combined with the GW method, which enables one to treat strong interaction effects [28]. One of the main features of the new scheme is that the Hubbard $U$ is calculated from first principles through a self-consistency requirement on the on-site screened Coulomb interaction, analogous to the self-consistency in the local Green's function in the DMFT. Since the GWA has an explicit diagrammatic representation, the on-site contribution of the GW self-energy can be readily identified and the scheme then allows for a precise double-counting correction.

In the next two sections, we will give a summary of the GWA and DMFT, describing 
their main features. In the fourth section we lay out the GW+DMFT scheme, followed by a simplified application of the scheme to the excitation spectrum of nickel. Finally we discuss some future challenges and directions.

\subsection{The $G W$ Approximation}

\subsubsection{Theory}

It can be shown that the self-energy may be expressed as [20]

$$
\Sigma(1,2)=-i \int d 3 d 4 v(1,4) G(1,3) \frac{\delta G^{-1}(3,2)}{\delta \phi(4)}
$$

where $v$ is the bare Coulomb interaction, $G$ is the Green function and $\phi$ is an external timedependent probing field. We have used the short-hand notation $1=\left(x_{1} t_{1}\right)$. From the equation of motion of the Green function

$$
\begin{aligned}
& G^{-1}=i \frac{\partial}{\partial t}-H_{0}-\Sigma \\
& H_{0}=h_{0}+\phi+V_{H}
\end{aligned}
$$

$h_{0}$ is the kinetic energy and $V_{H}$ is the Hartree potential. We then obtain

$$
\begin{aligned}
\frac{\delta G^{-1}(3,2)}{\delta \phi(4)} & =-\delta(3-2)\left[\delta(3-4)+\frac{\delta V_{H}(3)}{\delta \phi(4)}\right]-\frac{\delta \Sigma(3,2)}{\delta \phi(4)} \\
& =-\delta(3-2) \epsilon^{-1}(3,4)-\frac{\delta \Sigma(3,2)}{\delta \phi(4)}
\end{aligned}
$$

where $\epsilon^{1}$ is the inverse dielectric matrix. The GWA is obtained by neglecting the vertex correction $\delta \Sigma / \delta \phi$, which is the last term in (1.4). This is just the random-phase approximation (RPA) for $\epsilon^{-1}$. This leads to

$$
\Sigma(1,2)=i G(1,2) W(1,2)
$$

where we have defined the screened Coulomb interaction $W$ by

$$
W(1,2)=\int d 3 v(1,3) \epsilon^{-1}(3,2)
$$

The RPA dielectric function is given by

$$
\epsilon=1-v P
$$


where

$$
\begin{aligned}
P\left(\mathbf{r}, \mathbf{r}^{\prime} ; \omega\right) & =-2 i \int \frac{d \omega^{\prime}}{2 \pi} G\left(\mathbf{r}, \mathbf{r}^{\prime} ; \omega+\omega^{\prime}\right) G\left(\mathbf{r}^{\prime}, \mathbf{r} ; \omega^{\prime}\right) \\
& =2 \sum_{i} \sum_{j}^{\text {occ unocc }} \psi_{i}(\mathbf{r}) \psi_{i}^{*}\left(\mathbf{r}^{\prime}\right) \psi_{j}^{*}(\mathbf{r}) \psi_{j}\left(\mathbf{r}^{\prime}\right) \\
& \times\left\{\frac{1}{\omega-\varepsilon_{j}+\varepsilon_{i}+i \delta}-\frac{1}{\omega+\varepsilon_{j}-\varepsilon_{i}-i \delta}\right\}
\end{aligned}
$$

with the Green function constructed from a one-particle band structure $\left\{\psi_{i}, \varepsilon_{i}\right\}$. The factor of 2 arises from the sum over spin variables. In frequency space, the self-energy in the GWA takes the form

$$
\Sigma\left(r, r^{\prime} ; \omega\right)=\frac{i}{2 \pi} \int d \omega^{\prime} e^{i \eta \omega^{\prime}} G\left(r, r^{\prime} ; \omega+\omega^{\prime}\right) W\left(r, r^{\prime} ; \omega^{\prime}\right)
$$

We have so far described the zero temperature formalism. For finite temperature we have

$$
\begin{aligned}
& P\left(\mathbf{r}, \mathbf{r}^{\prime} ; i \nu_{n}\right)=\frac{2}{\beta} \sum_{\omega_{k}} G\left(\mathbf{r}, \mathbf{r}^{\prime} ; i \nu_{n}+i \omega_{k}\right) G\left(\mathbf{r}^{\prime}, \mathbf{r} ; i \omega_{k}\right) \\
& \Sigma\left(r, r^{\prime} ; i \omega_{n}\right)=-\frac{1}{\beta} \sum_{\nu_{k}} G\left(r, r^{\prime} ; i \omega_{n}+i \nu_{k}\right) W\left(r, r^{\prime} ; i \nu_{k}\right)
\end{aligned}
$$

In the Green function language, the Fock exchange operator in the Hartree-Fock approximation (HFA) can be written as $i G v$. We may therefore regard the GWA as a generalization of the HFA, where the bare Coulomb interaction $v$ is replaced by a screened interaction $W$. We may also think of the GWA as a mapping to a polaron problem where the electrons are coupled to some bosonic excitations (e.g., plasmons) and the parameters in this model are obtained from first-principles calculations.

The replacement of $v$ by $W$ is an important step in solids where screening effects are generally rather large relative to exchange, especially in metals. For example, in the electron gas, within the GWA exchange and correlation are approximately equal in magnitude, to a large extent cancelling each other, modifying the free-electron dispersion slightly. But also in molecules, accurate calculations of the excitation spectrum cannot neglect the effects of correlations or screening. The GWA is physically sound because it is qualitatively correct in some limiting cases [21].

\subsubsection{The $\mathbf{G W}$ approximation in practice}

The quality of the GWA may be seen in Figure (1.1), where a plot of band gaps of a number of well known semiconductors and insulators is displayed. It is clear from the plot that the LDA 
Band gaps of semiconductors and insulators

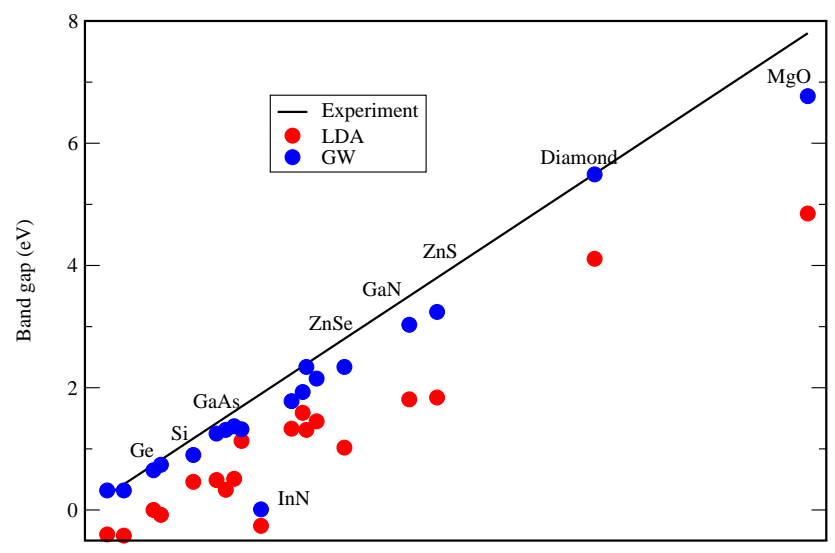

Figure 1.1: Band gaps of some selected semiconductors and insulators calculated within the GWA compared with the LDA and experimental values. The GW data are taken from [26].

systematically underestimates the band gaps and that the GWA substantially improves the LDA band gaps. It has been found that for some materials, like $\mathrm{MgO}$ and $\mathrm{InN}$, significant error still remains within the GWA. The reason for the discrepancy has not been understood well. One possible explanation is that the result of the one-iteration GW calculation may depend on the starting one-particle band structure. For example, in the case of InN, the starting LDA band structure has no gap. This may produce a metal-like (over)screened interaction $W$ which fails to open up a gap or yields too small gap in the $\mathrm{GW}$ calculation. A similar behaviour is also found in the more extreme case of $\mathrm{NiO}$, where a one-iteration $\mathrm{GW}$ calculation only yields a gap of about $1 \mathrm{eV}$ starting from an LDA gap of $0.2 \mathrm{eV}$ (the experimental gap is $4 \mathrm{eV}$ ) [9 [10].

The problems with the GWA arise when it is applied to strongly correlated systems. Application to ferromagnetic nickel [8] illustrates some of the difficulties with the GWA. Starting from the LDA band structure, a one-iteration GW calculation does improve significantly the LDA band structure. In particular it reduces the too large $3 \mathrm{~d}$ band width bringing it into much better agreement with photoemission data. However, the too large LDA exchange splitting $(0.6 \mathrm{eV}$ compared with experimental value of $0.3 \mathrm{eV}$ ) remains essentially unchanged. Moreover, the famous $6 \mathrm{eV}$ satellite, which is of course missing in the LDA, is not reproduced. These problems point to deficiencies in the GWA in describing short-range correlations since we expect that both exchange splitting and satellite structure are influenced by on-site interactions. In the case of exchange splitting, long-range screening also plays a role in reducing the HF value and the problem with the exchange splitting indicates a lack of spin-dependent interaction in the GWA: In the GWA the spin dependence only enters in $G$ but not in $W$.

Application to NiO, the prototype of Mott-Hubbard transition metal oxides, reveals another difficulty with the one-iteration GWA. As already mentioned previously, when the starting band structure is far from the experimental quasiparticle band structure, a one-iteration GW calculation may not be sufficient. This problem may be circumvented by performing 
a partial self-consistent calculation in which the self-energy from the previous iteration at a given energy, such as the Fermi energy of the centre of the band of interest, is used to construct a new set of one-particle orbitals. This procedure is continued to self-consistency such that the starting one-particle band structure gives zero self-energy correction [9, 10, 16]. A more serious problem, however, is describing the charge-transfer character of the top of the valence band. The GWA essentially still maintains the Mott-Hubbard band gap as in the LDA, i.e., the top of the valence band is mainly of $3 \mathrm{~d}$ character rather than the charge-transfer character dominated by the $2 \mathrm{p}$ oxygen hole. As in nickel, the problem with the satellite arises again. Depending on the starting band structure, a satellite may be reproduced albeit at a too high energy. Thus there is a strong need for improving the short-range correlations in the GWA which may be achieved by using a suitable approach based on the dynamical mean-field theory described in the next section.

\subsection{Dynamical Mean Field Theory}

Dynamical mean field theory (DMFT) [19] has originally been developed within the context of models for correlated fermions on a lattice where it has proven very successful for determining the phase diagrams or for calculations of excited states properties. It is a nonperturbative method and as such appropriate for systems with any strength of the interaction. In recent years, combinations of DMFT with band structure theory, in particular Density functional theory with the local density approximation (LDA) have emerged. The idea is to correct for shortcomings of DFT-LDA due to strong Coulomb interactions and localization (or partial localization) phenomena that cause effects very different from a homogeneous itinerant behaviour. Such signatures of correlations are well-known in transition metal oxides or felectron systems but are also present in several elemental transition metals.

The application of DMFT to real solids relies on a representability conjecture assuming that local quantities such as for example the local Green's function or self-energy of a solid can be calculated from a local impurity model, that is one can find a dynamical mean field $\mathcal{G}_{0}$ and a Hubbard parameter $U$, such that the Green's function calculated from the effective action

$$
\begin{aligned}
S & =\int_{0}^{\beta} d \tau \sum_{m \sigma} c_{m \sigma}^{\dagger}(\tau) \mathcal{G}_{0 m m^{\prime} \sigma}^{-1}\left(\tau-\tau^{\prime}\right) c_{m^{\prime} \sigma}\left(\tau^{\prime}\right) \\
& +\frac{1}{2} \int_{0}^{\beta} d \tau \sum_{m m^{\prime} \sigma} U_{m m^{\prime}} n_{m \sigma}(\tau) n_{m^{\prime}-\sigma}(\tau) \\
& +\frac{1}{2} \int_{0}^{\beta} d \tau \sum_{m \neq m^{\prime} \sigma}\left(U_{m m^{\prime}}-J_{m m^{\prime}}\right) n_{m \sigma}(\tau) n_{m^{\prime} \sigma}(\tau)
\end{aligned}
$$

coincides with the local Green's function of the solid. For a model of correlated fermions on a lattice with infinite coordination number this conjecture can rigorously be proven: it is a consequence of the absence of any non-local contributions to the self-energy of the system. For a real solid the situation is somewhat more complicated, not only due to the finite coordination number but also to the difficulty to define the notion of locality. This notion is 
crucial at both stages, for the construction of the impurity model, where long-range Coulomb interactions are mimicked by local Hubbard parameters ${ }^{2}$, and for the resolution of the model within DMFT, which approximates the full self-energy of the model by a local quantity. Applications of DMFT to electronic structure calculations (e.g. the LDA+DMFT method) are therefore always defined within a specific basis set using localized basis functions. Within an LMTO implementation for example locality can naturally be defined as referring to the same muffin tin sphere. This amounts to defining matrix elements $G_{L \mathbf{R}, L^{\prime} \mathbf{R}^{\prime}}(i \omega)$ of the full Green's function

$$
G\left(\mathbf{r}, \mathbf{r}^{\prime}, i \omega\right)=\sum_{L L^{\prime} \mathbf{R} \mathbf{R}^{\prime}} \chi_{L \mathbf{R}}^{*}(\mathbf{r}) G_{L \mathbf{R}, L^{\prime} \mathbf{R}^{\prime}}(i \omega) \chi_{L^{\prime} \mathbf{R}^{\prime}}\left(\mathbf{r}^{\prime}\right)
$$

and assuming that its local, that is "on-sphere" part equals the Green's function of the local impurity model (1.13). Here $\mathbf{R}, \mathbf{R}^{\prime}$ denote the coordinates of the centres of the muffin tin spheres, while $\mathbf{r}, \mathbf{r}^{\prime}$ can take any values. The index $L=(n, l, m)$ regroups all radial and angular quantum numbers. The dynamical mean field $\mathcal{G}_{0}$ in (1.13) has to be determined in such a way that the Green's function $G_{\text {impurity } L, L^{\prime}, \sigma}$ of the impurity model Eq.11.13 coincides with $G_{L \mathbf{R}, L^{\prime} \mathbf{R}^{\prime}}(i \omega)$ if the impurity model self-energy is used as an estimate for the true selfenergy of the solid. This self-consistency condition reads

$$
G_{\text {impurity }}\left(i \omega_{n}\right)=\sum_{k}\left(i \omega_{n}+\mu-H_{o}(k)-\Sigma\left(i \omega_{n}\right)\right)^{-1}
$$

where $\Sigma, H_{0}$ and $G$ are matrices in orbital and spin space, and $i \omega+\mu$ is a matrix proportional to the unit matrix in that space.

Together with (1.13) this defines the DMFT equations that have to be solved self-consistently. Note that the main approximation of DMFT is hidden in the self-consistency condition where the local self-energy has been promoted to the full lattice self-energy.

The representability assumption can actually be extended to other quantities of a solid than its local Green's function and self-energy. In "extended DMFT" 44, 27, 24 43] e.g. a two particle correlation function is calculated and can then be used in order to represent the local screened Coulomb interaction $W$ of the solid. This is the starting point of the "GW+DMFT" scheme described in section 6 .

\subsubsection{DMFT in practice}

Combinations of DFT-LDA and DMFT, so-called "LDA+DMFT" techniques [7], have so far been applied to transition metals (Fe, Ni, Mn) and their oxides (e.g. $\mathrm{La} / \mathrm{YTiO}_{3}, \mathrm{~V}_{2} \mathrm{O}_{3}$, $\left.\mathrm{Sr} / \mathrm{CaVO}_{3}\right)$ as well as elemental $\mathrm{f}$-electron materials $(\mathrm{Pu}, \mathrm{Ce})$ and their compounds. In the

\footnotetext{
${ }^{2}$ For a discussion of the appropriateness of local Hubbard parameters see [11.
} 
most general formulation, one starts from a many-body Hamiltonian of the form

$$
\begin{aligned}
H & =\sum_{\{i m \sigma\}}\left(H_{i m, i^{\prime} m^{\prime}}^{L D A}-H^{d c}\right) a_{i m \sigma}^{+} a_{i^{\prime} m^{\prime} \sigma} \\
& +\frac{1}{2} \sum_{i m m^{\prime} \sigma} U_{m m^{\prime}}^{i} n_{i m \sigma} n_{i m^{\prime}-\sigma} \\
& +\frac{1}{2} \sum_{i m \neq m^{\prime} \sigma}\left(U_{m m^{\prime}}^{i}-J_{m m^{\prime}}^{i}\right) n_{i m \sigma} n_{i m^{\prime} \sigma}
\end{aligned}
$$

where $H^{L D A}$ is the effective Kohn-Sham-Hamiltonian derived from a self-consistent DFTLDA calculation. This one-particle Hamiltonian is then corrected by Hubbard terms for direct and exchange interactions for the "correlated" orbitals, e.g. $d$ or $f$ orbitals. In order to avoid double counting of the Coulomb interactions for these orbitals, a correction term $H^{d c}$ is subtracted from the original LDA Hamiltonian. The resulting Hamiltonian (1.14) is then treated within dynamical mean field theory by assuming that the many-body self-energy associated with the Hubbard interaction terms can be calculated from a multi-band impurity model.

This general scheme can be simplified in specific cases, e.g. in systems with a separation of the correlated bands from the "uncorrelated" ones, an effective model of the correlated bands can be constructed; symmetries of the crystal structure can be used to reduce the number of components of the self-energy etc.

Despite the huge progress made in the understanding of the electronic structure of correlated materials thanks to such LDA+DMFT schemes, certain conceptual problems remain open: These are related to the choice of the Hubbard interaction parameters and to the double counting corrections. An a priori choice of which orbitals are treated as correlated and which orbitals are left uncorrelated has to be made, and the values of $U$ and $J$ have to be fixed. Attempts of calculating these parameters from constrained LDA techniques are appealing in the sense that one can avoid introducing external parameters to the theory, but suffer from the conceptual drawback in that screening is taken into account in a static manner only [11]. Finally, the double counting terms are necessarily ill defined due to the impossibility to single out in the LDA treatment contributions to the interactions stemming from specific orbitals. These drawbacks of LDA+DMFT provide a strong motivation to attempt the construction of an electronic structure method for correlated materials beyond combinations of LDA and DMFT.

\subsection{GW+DMFT}

The idea behind combining the GWA and the DMFT is to take advantage of the strong features of the two theories. The GWA, being based on RPA screening, is capable of taking into account long-range correlations but does not describe properly short-range correlations. On the other hand, the strength of the DMFT is its ability to describe on-site correlations by means of a mapping of the many-body problem onto an impurity problem where the onsite interactions are summed to all orders. While the GWA is a fully first-principles theory, the DMFT has been traditionally used in conjunction with a Hubbard model. Clearly, it is 
desirable to combine the two theories into a consistent theory where the parameters in the Hubbard model are determined self-consistently from first-principles via the GWA.

Recently, first steps have been undertaken towards a combination of the GWA and DMFT, both in a model context [46] and within the framework of realistic electronic structure calculations [13]. The basic physical idea of GW+DMFT is to separate the lattice into an on-site part and the rest. The on-site self-energy is taken to be the impurity self-energy calculated by the DMFT and the off-site self-energy is calculated by the GWA. Viewed from the GWA, we replace the on-site GW self-energy by that of DMFT, correcting the GW treatment of onsite correlations. Viewed from the DMFT, we add off-site contributions to the self-energy approximated within the GWA, giving a momentum dependent self-energy.

The impurity problem contains a Hubbard interaction $U$ that is usually treated as a parameter. In order to calculate the $\mathrm{U}$, we introduce a self-consistency condition that the $\mathrm{U}$ screened by the effective bath in the impurity model be equal to the local projection of the global screened interaction $\mathrm{W}$. This condition is complementary to the condition imposed in the DMFT that the impurity Green's function be equal to the local Green's function.

The above physical ideas can be formulated in a precise way using the free-energy functional of Luttinger and Ward (LW). A generalization of the original LW functional takes the form [1](see also [15])

$$
\Gamma(G, W)=\operatorname{Tr} \ln G-\operatorname{Tr}\left[\left(G_{H}^{-1}-G^{-1}\right) G\right]-\frac{1}{2} \operatorname{Tr} \ln W+\frac{1}{2} \operatorname{Tr}\left[\left(v^{-1}-W^{-1}\right) W\right]+\Psi[G, W]
$$

$G_{H}^{-1}=i \omega_{n}+\mu+\nabla^{2} / 2-V_{H}$ corresponds to the Hartree Green's function with $V_{H}$ being the Hartree potential. The functional $\Psi[G, W]$ is a generalization of the original LW $\Phi[G]$ functional, whose derivative with respect to $G$ gives the self-energy. A more general derivation of (1.15) using a Hubbard-Stratonovich transformation and a Legendre transformation with respect to both $G$ and $W$ may be found in a later work [15]. It is straightforward to verify that at equilibrium the stationarity of $\Gamma$ yields

$$
\begin{aligned}
& \frac{\delta \Gamma}{\delta G}=0 \rightarrow G^{-1}=G_{H}^{-1}-\Sigma, \quad \Sigma=\frac{\delta \Psi}{\delta G} \\
& \frac{\delta \Gamma}{\delta W}=0 \rightarrow W^{-1}=v^{-1}-P, \quad P=-2 \frac{\delta \Psi}{\delta W}
\end{aligned}
$$

The functional $\Psi$ is divided into on-site and off-site components:

$$
\Psi=\Psi_{G W}^{o f f-s i t e}\left[G^{R R^{\prime}}, W^{R R^{\prime}}\right]+\Psi_{i m p}^{o n-s i t e}\left[G^{R R}, W^{R R}\right]
$$

where $R$ denotes a lattice site. In the GWA the functional $\Psi$ is given by

$$
\Psi_{G W}[G, W]=\frac{1}{2} G W G
$$


The impurity part $\Psi_{i m p}$ is generated from a local quantum impurity problem defined on a single atomic site with an effective action

$$
\begin{aligned}
S & =\int d \tau d \tau^{\prime}\left[-\sum c_{L}^{+}(\tau) \mathcal{G}_{L L^{\prime}}^{-1}\left(\tau-\tau^{\prime}\right) c_{L^{\prime}}\left(\tau^{\prime}\right)\right. \\
& \left.+\frac{1}{2} \sum: c_{L_{1}}^{+}(\tau) c_{L_{2}}(\tau): \mathcal{U}_{L_{1} L_{2} L_{3} L_{4}}\left(\tau-\tau^{\prime}\right): c_{L_{3}}^{+}\left(\tau^{\prime}\right) c_{L_{4}}\left(\tau^{\prime}\right):\right]
\end{aligned}
$$

The double dots denote normal ordering and $L$ refers to an orbital of angular momentum $L$ on a given sphere where the impurity problem is defined. These orbitals are usually partially filled localized $3 \mathrm{~d}$ or $4 \mathrm{f}$ orbitals.

The GW+DMFT set of equations can now be readily derived from 1.15, 1.18, and 1.19 by taking functional derivatives of $\Psi$ with respect to $G$ and $W$ as in 1.16 and (1.17):

$$
\begin{aligned}
& \Sigma=\Sigma_{G W}^{R R^{\prime}}\left(1-\delta_{R R^{\prime}}\right)+\Sigma_{i m p}^{R R} \delta_{R R^{\prime}} \\
& P=P_{G W}^{R R^{\prime}}\left(1-\delta_{R R^{\prime}}\right)+P_{i m p}^{R R} \delta_{R R^{\prime}}
\end{aligned}
$$

In practice the self-energy is expanded in some basis set $\left\{\phi_{L}\right\}$ localized in a site. The polarization function on the other hand is expanded in a set of two-particle basis functions $\left\{\phi_{L} \phi_{L^{\prime}}\right\}$ (product basis) since the polarization corresponds to a two-particle propagator. For example, when using the linear muffin-tin orbital (LMTO) band-structure method, the product basis consists of products of LMTO's. These product functions are generally linearly dependent and a new set of optimized product basis (OPB) [10] is constructed by forming linear combinations of product functions, eliminating the linear dependencies. We denote the OPB set by $B_{\alpha}=\sum_{L L^{\prime}} \phi_{L} \phi_{L^{\prime}} c_{L L^{\prime}}^{\alpha}$. To summarize, one-particle quantities like $G$ and $\Sigma$ are expanded in $\left\{\phi_{L}\right\}$ whereas two-particle quantities such as $P$ and $W$ are expanded in the OPB set $\left\{B_{\alpha}\right\}$. It is important to note that the number of $\left\{B_{\alpha}\right\}$ is generally smaller than the number of $\left\{\phi_{L} \phi_{L^{\prime}}\right\}$ so that quantities expressed in $\left\{B_{\alpha}\right\}$ can be expressed in $\left\{\phi_{L} \phi_{L^{\prime}}\right\}$, but not vice versa. In momentum space, equations 1.21 and 1.22 read

$$
\begin{aligned}
& \Sigma^{L L^{\prime}}\left(\mathbf{k}, i \omega_{n}\right)=\Sigma_{G W}^{L L^{\prime}}\left(\mathbf{k}, i \omega_{n}\right)-\sum_{\mathbf{k}} \Sigma_{G W}^{L L^{\prime}}\left(\mathbf{k}, i \omega_{n}\right)+\Sigma_{i m p}^{L L^{\prime}}\left(i \omega_{n}\right) \\
& P^{\alpha \beta}\left(\mathbf{k}, i \omega_{n}\right)=P_{G W}^{\alpha \beta}\left(\mathbf{k}, i \omega_{n}\right)-\sum_{\mathbf{k}} P_{G W}^{\alpha \beta}\left(\mathbf{k}, i \omega_{n}\right)+P_{i m p}^{\alpha \beta}\left(i \omega_{n}\right)
\end{aligned}
$$

The second terms in the above two equations remove the on-site contributions of the GW self-energy and polarization, which are already included in $\Sigma_{i m p}$ and $P_{i m p}$.

We are now in a position to outline the self-consistency loop which determines $\mathcal{G}$ and $\mathcal{U}$ as well as the full $G$ and $W$ self-consistently. 
- The impurity problem (1.20) is solved, for a given choice of Weiss field $\mathcal{G}_{L L^{\prime}}$ and Hubbard interaction $\mathcal{U}_{\alpha \beta}$ : the "impurity" Green's function

$$
G_{i m p}^{L L^{\prime}} \equiv-\left\langle T_{\tau} c_{L}(\tau) c_{L^{\prime}}^{+}\left(\tau^{\prime}\right)\right\rangle_{S}
$$

is calculated, together with the impurity self-energy

$$
\Sigma_{i m p} \equiv \delta \Psi_{i m p} / \delta G_{i m p}=\mathcal{G}^{-1}-G_{i m p}^{-1} .
$$

The two-particle correlation function

$$
\chi_{L_{1} L_{2} L_{3} L_{4}}=\left\langle: c_{L_{1}}^{\dagger}(\tau) c_{L_{2}}(\tau):: c_{L_{3}}^{\dagger}\left(\tau^{\prime}\right) c_{L_{4}}\left(\tau^{\prime}\right):\right\rangle_{S}
$$

must also be evaluated.

- The impurity effective interaction is constructed as follows:

$$
W_{i m p}^{\alpha \beta}=\mathcal{U}_{\alpha \beta}-\sum_{L_{1} \cdots L_{4}} \sum_{\gamma \delta} \mathcal{U}_{\alpha \gamma} O_{L_{1} L_{2}}^{\gamma} \chi_{L_{1} L_{2} L_{3} L_{4}}\left[O_{L_{3} L_{4}}^{\delta}\right]^{*} \mathcal{U}_{\delta \beta}
$$

Here all quantities are evaluated at the same frequency ${ }^{3}$ and $O_{L_{1} L_{2}}^{\gamma}$ is the overlap matrix $\left\langle B_{\gamma} \mid \phi_{L} \phi_{L^{\prime}}\right\rangle$. The polarization operator of the impurity problem is then obtained as:

$$
P_{i m p} \equiv-2 \delta \Psi_{i m p} / \delta W_{i m p}=\mathcal{U}^{-1}-W_{i m p}^{-1},
$$

where the matrix inversions are performed in the OPB $\left\{B_{\alpha}\right\}$.

- From Eqs. (1.23) and (1.24) the full k-dependent Green's function $G\left(\mathbf{k}, i \omega_{n}\right)$ and effective interaction $W\left(\mathbf{q}, i \nu_{n}\right)$ can be constructed. The self-consistency condition is obtained, as in the usual DMFT context, by requiring that the on-site components of these quantities coincide with $G_{i m p}$ and $W_{i m p}$. In practice, this is done by computing the on-site quantities

$$
\begin{aligned}
G_{l o c}\left(i \omega_{n}\right) & =\sum_{\mathbf{k}}\left[G_{H}^{-1}\left(\mathbf{k}, i \omega_{n}\right)-\Sigma\left(\mathbf{k}, i \omega_{n}\right)\right]^{-1} \\
W_{l o c}\left(i \nu_{n}\right) & =\sum_{\mathbf{q}}\left[V_{\mathbf{q}}^{-1}-P\left(\mathbf{q}, i \nu_{n}\right)\right]^{-1}
\end{aligned}
$$

and using them to update the Weiss dynamical mean field $\mathcal{G}$ and the impurity model interaction $\mathcal{U}$ according to:

$$
\begin{aligned}
\mathcal{G}^{-1} & =G_{l o c}^{-1}+\Sigma_{i m p} \\
\mathcal{U}^{-1} & =W_{l o c}^{-1}+P_{i m p}
\end{aligned}
$$

\footnotetext{
${ }^{3}$ Note that $\chi_{L_{1} \ldots L_{4}}$ does not denote the matrix element $\left\langle L_{1} L_{2}|\chi| L_{3} L_{4}\right\rangle$, but is rather defined by $\chi\left(r, r^{\prime}\right)=$ $\sum_{L_{1} \ldots L_{4}} \phi_{L_{1}}^{*}(r) \phi_{L_{2}}^{*}(r) \chi_{L_{1} \ldots L_{4}} \phi_{L_{3}}\left(r^{\prime}\right) \phi_{L_{4}}\left(r^{\prime}\right)$.
} 
This cycle is iterated until self-consistency for $\mathcal{G}$ and $\mathcal{U}$ is obtained (as well as on $G, W$, $\Sigma^{x c}$ and $P$ ). When self-consistency is reached, $G_{i m p}=G_{l o c}$ and $W_{i m p}=W_{l o c}$. This implies that at self-consistency, the second term in (1.23) can be rewritten as (in imaginary-time)

$$
\sum_{\mathbf{k}} \Sigma_{G W}^{L L^{\prime}}(\mathbf{k}, \tau)=-\sum_{L_{1} L_{1}^{\prime}} W_{i m p}^{L L_{1} L^{\prime} L_{1}^{\prime}}(\tau) G_{i m p}^{L_{1}^{\prime} L_{1}}(\tau)
$$

This shows that the local or on-site contribution of the GW self-energy is precisely subtracted out, thus avoiding double counting. Eventually, self-consistency over the local electronic density can also be implemented, (in a similar way as in LDA+DMFT [40, 41]) by recalculating $\rho(\boldsymbol{r})$ from the Green's function at the end of the convergence cycle above, and constructing an updated Hartree potential. This new density is used as an input of a new GW calculation, and convergence over this external loop must be reached. While implementing self-consistency within the GWA is known to yield unsatisfactory spectra [23], we expect a more favourable situation in the proposed GW+DMFT scheme since part of the interaction effects are treated to all orders.

\subsubsection{Simplified implementation of GW+DMFT and application to ferromagnetic nickel}

The full implementation of the proposed approach in a fully dynamical and self-consistent manner is at the present stage computationally very demanding and we regard it as a major challenge for future research. Here, we apply a simplified scheme of the approach [13] to the electronic structure of nickel in order to demonstrate its feasibility and potential. The main simplifications made are:

1. The DMFT local treatment is applied only to the $d$-orbitals, and we replace the dynamical impurity problem by its static limit, solving the impurity model 1.20 for a frequencyindependent $\mathcal{U}=\mathcal{U}(\omega=0)$.

2. We perform a one-iteration GW calculation in the form [10]: $\Sigma_{G W}=G_{L D A} \cdot W\left[G_{L D A}\right]$, from which the off-site part of the self-energy is obtained.

The local Green's function is taken to be

$$
\begin{aligned}
G_{l o c}^{\sigma}\left(i \omega_{n}\right) & =\sum_{\mathbf{k}}\left[G_{H}^{-1}\left(\mathbf{k}, i \omega_{n}\right)-\Sigma_{G W}^{o f f-s i t e}\right. \\
& \left.-\left(\Sigma_{i m p, \sigma}-\frac{1}{2} \operatorname{Tr}_{\sigma} \Sigma_{i m p, \sigma}(0)+V_{x c}^{o n-s i t e}\right)\right]^{-1}
\end{aligned}
$$

Thus, the off-site part is obtained from the GW self-energy whereas the on-site part is derived from the impurity self-energy with a double-counting correction of the form proposed in [32].

We have performed finite temperature GW and LDA+DMFT calculations (within the LMTO-ASA [2] with 29 irreducible k-points) for ferromagnetic nickel (lattice constant 6.654 a.u.), using $4 \mathrm{~s} 4 \mathrm{p} 3 \mathrm{~d} 4 \mathrm{f}$ states, at the Matsubara frequencies $i \omega_{n}$ corresponding to $T=630 \mathrm{~K}$, just below the Curie temperature. The GW self-energy is calculated from a paramagnetic 


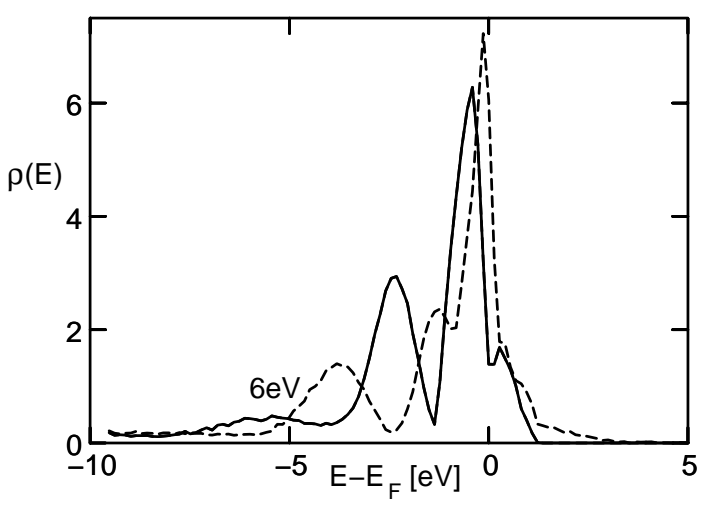

Figure 1.2: Partial density of states pf $3 \mathrm{~d}$ orbitals of nickel (solid/dashed lines give the majority/minority spin contribution) as obtained from the combination of GW and DMFT [13]. For comparison with LDA and LDA+DMFT results, see [32]; for experimental spectra, see [35].

Green's function, leaving the spin-dependence to the impurity self-energy. The resulting selfenergies are inserted into Eq. 1.35, which is then used to calculate a new Weiss field according to 1.32. The Green's function $G_{l o c}^{\sigma}(\tau)$ is recalculated from the impurity effective action by QMC and analytically continued using the Maximum Entropy algorithm. The resulting spectral function is plotted in Fig.(1.2). Comparison with the LDA+DMFT results in [32] shows that the good description of the satellite structure, exchange splitting and band narrowing is indeed retained within the (simplified) GW+DMFT scheme. We have also calculated the quasiparticle band structure, from the poles of [1.35], after linearization of $\Sigma\left(\mathbf{k}, i \omega_{n}\right)$ around the Fermi level ${ }^{4}$. Fig. (1.3) shows a comparison of GW+DMFT with the LDA and experimental band structure. It is seen that GW+DMFT correctly yields the bandwidth reduction compared to the (too large) LDA value and renormalizes the bands in a (k-dependent) manner.

Because of the static approximation 3), we could not implement self-consistency on $W_{l o c}$ (Eq. 1.31). We chose the value of $\mathcal{U}(\omega=0)(\simeq 3.2 \mathrm{eV})$ by calculating the correlation function $\chi$ and ensuring that Eq. (1.28) is fulfilled at $\omega=0$, given the GW value for $W_{l o c}(\omega=$ $0)(\simeq 2.2 \mathrm{eV}$ for Nickel [45]).

\subsection{Conclusions}

The proposed GW+DMFT scheme avoids the conceptual problems inherent to "LDA+DMFT" methods, such as double counting corrections and the use of Hubbard parameters assigned to correlated orbitals. The notion of self-consistency condition on the on-site Green's function in the DMFT is extended to the screened interaction. Analogous to the usual condition that the

\footnotetext{
${ }^{4}$ Note however that this linearization is no longer meaningful at energies far away from the Fermi level. We therefore use the unrenormalized value for the quasi-particle residue for the s-band $\left(Z_{s}=1\right)$.
} 


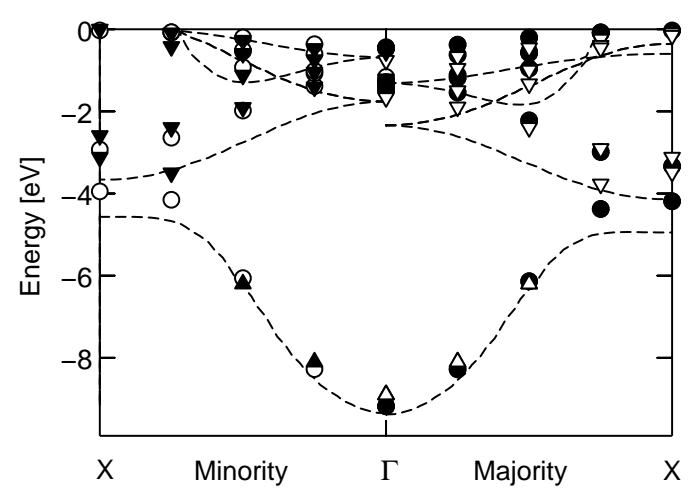

Figure 1.3: Band structure of nickel (majority and minority spins) from GW+DMFT scheme [13] (circles) in comparison to the LDA band structure (dashed lines) and experiments [14] (triangles down) and [35] (triangles up).

impurity Green's function be equal to the on-site Green's function, we demand that the on-site screened Hubbard $\mathcal{U}$ of the impurity be equal to the on-site projection of the global screened interaction $W$. In this fashion, the Hubbard $\mathcal{U}$ is determined from first-principles. Since the GWA has a well-defined diagrammatic interpretation, it is also possible to precisely take into account the double-counting correction.

A number of issues are of immediate importance. Solving impurity models with frequencydependent interaction parameters [34 17, 46] is one of the most urgent tasks as well as studies of various possible self-consistency schemes. To study these aspects, we are now carrying out GW+DMFT calculations on the electron gas. Applications to real materials are both theoretically and computationally very challenging. Extension to the two-particle Green's function is another field for future research [37].

\subsection{Acknowledgments}

This work was supported in part by NAREGI Nanoscience Project, Ministry of Education, Culture, Sports, Science and Technology, Japan and by a grant of supercomputing time at IDRIS Orsay, France (project number 031393). 



\section{Bibliography}

[1] C.-O. Almbladh, U. von Barth and R. van Leeuwen, Int. J. Mod. Phys. B 13, 535 (1999)

[2] O. K. Andersen, Phys. Rev. B 12, 3060 (1975); O. K. Andersen, T. Saha-Dasgupta, S. Erzhov, Bull. Mater. Sci. 26, 19 (2003)

[3] V. I. Anisimov, J. Zaanen, and O. K. Andersen, Phys. Rev. B 44, 943 (1991)

[4] V. I. Anisimov, I. V. Solovyev, M. A. Korotin, M. T. Czyzyk, and G. A. Sawatzky, Phys. Rev. B 48, 16929 (1993)

[5] For reviews, see V. I. Anisimov, F. Aryasetiawan, and A. I. Lichtenstein, J. Phys.: Condens. Matter 9, 767 (1997)

[6] V. I. Anisimov et al., J. Phys.: Condens. Matter 9, 7359 (1997)

[7] For reviews, see Strong Coulomb correlations in electronic structure calculations, edited by V. I. Anisimov, Advances in Condensed Material Science (Gordon and Breach, New York, 2001)

[8] F. Aryasetiawan, Phys. Rev. B 46, 13051 (1992)

[9] F. Aryasetiawan and O. Gunnarsson, Phys. Rev. Lett. 74, 3221 (1995)

[10] F. Aryasetiawan and O. Gunnarsson, Rep. Prog. Phys. 61, 237 (1998)

[11] F. Aryasetiawan et al., in preparation.

[12] W. G. Aulbur, L. Jönsson, and J. W. Wilkins, Solid State Physics 54, 1 (2000)

[13] S. Biermann, F. Aryasetiawan, and A. Georges, Phys. Rev. Lett. 90, 086402 (2003)

[14] J. Bünemann et al, Europhys. Lett. 61, 667 (2003)

[15] R. Chitra and G. Kotliar, Phys. Rev. B 63, 115110 (2001)

[16] S. V. Faleev, M. van Schilfgaarde, and T. Kotani, unpublished

[17] J. K. Freericks, M. Jarrell and D. J. Scalapino, Phys. Rev. B 48, 6302 (1993)

[18] A. Fujimori, F. Minami, and S. Sugano, Phys. Rev. B 29, 5225 (1984)

[19] For reviews, see A. Georges, G. Kotliar, W. Krauth, and M. J. Rosenberg, Rev. Mod. Phys. 68, 13 (1996); T. Pruschke et al, Adv. Phys. 44, 187 (1995)

[20] L. Hedin, Phys. Rev. 139, A796 (1965); L. Hedin and S. Lundqvist, Solid State Physics vol. 23, eds. H. Ehrenreich, F. Seitz, and D. Turnbull (Academic, New York, 1969)

[21] L. Hedin, Int. J. Quantum Chem. 54, 445 (1995)

[22] P. Hohenberg and W. Kohn, Phys. Rev. 136, B864 (1964)

[23] B. Holm and U. von Barth, Phys. Rev. B 57, 2108 (1998)

[24] H. Kajueter, Ph.D. thesis, Rutgers University, 1996

[25] W. Kohn and L. J. Sham, Phys. Rev. 140, A1133 (1965)

[26] T. Kotani and M. van Schilfgaarde, Solid State Communications 121, 461 (2002) 
[27] G. Kotliar and H. Kajueter (unpublished)

[28] For related ideas, see G. Kotliar and S. Savrasov, in New Theoretical Approaches to Strongly Correlated Systems, edited by A. M. Tsvelik (Kluwer Academic Publishing, Dordrecht, 2001) (and the updated version: cond-mat/0208241)

[29] W. Ku, A. G. Eguiluz, and E. W. Plummer, Phys. Rev. Lett. 85, 2410 (2000); H. Yasuhara, S. Yoshinaga, and M. Higuchi, ibid. 85, 2411 (2000)

[30] A. I. Lichtenstein, J. Zaanen, and V. I. Anisimov, Phys. Rev. B 52, R5467 (1995)

[31] A. I. Lichtenstein and M. I. Katsnelson, Phys. Rev. B 57, 6884 (1998).

[32] A. I. Lichtenstein, M. I. Katsnelson and G. Kotliar, Phys. Rev. Lett. 87, 067205 (2001)

[33] R. Maezono, M. D. Towler, Y. Lee, and R. J. Needs, Phys. Rev. B 68, 165103 (2003)

[34] Y. Motome and G. Kotliar, Phys. Rev. B 62, 12800 (2000)

[35] H. Mårtensson and P. O. Nilsson, Phys. Rev. B 30, 3047 (1984)

[36] J. E. Northrup, M. S. Hybertsen, and S. G. Louie, Phys. Rev. Lett. 59, 819 (1987); ibid. Phys. Rev. B 39, 8198 (1989)

[37] G. Onida, L. Reining and A. Rubio, Rev. Mod. Phys. 74, 601 (2002)

[38] See, e.g., J. P. Perdew, K. Burke, and M. Ernzerhof, Phys. Rev. Lett. 77, 3865 (1996) and references therein.

[39] See, e.g., W. E. Pickett, Rev. Mod. Phys. 62, 433 (1989)

[40] S. Savrasov and G. Kotliar,cond-mat/0106308

[41] S. Savrasov, G. Kotliar and E. Abrahams, Nature (London) 410, 793 (2000)

[42] G. A. Sawatzky and J. W. Allen, Phys. Rev. Lett. 53, 2339 (1984)

[43] A. M. Sengupta and A. Georges, Phys. Rev. B 52, 10295 (1995)

[44] Q. Si and J. L. Smith, Phys. Rev. Lett. 77, 3391 (1996)

[45] M. Springer and F. Aryasetiawan, Phys. Rev. B 57, 4364 (1998)

[46] P. Sun and G. Kotliar, Phys. Rev. B 66, 085120 (2002)

[47] M. P. Surh, J. E. Northrup, and S. G. Louie, Phys. Rev. B 38, 5976 (1988)

[48] Y. Takada, Phys. Rev. Lett. 87, 226402 (2001)

[49] L. H. Tjeng, C. T. Chen, J. Ghijsen, P. Rudolf, and F. Sette, Phys. Rev. Lett. 34, 501 (1991)

[50] H. Yasuhara, S. Yoshinaga and M. Higuchi, Phys. Rev. Lett. 83, 3250 (1999) 